Voraussetzung sein. Eine Therapie mit diesem Arzneimitte kann haftungs- und sozialrechtlich geboten sein, ist aber faktisch erst dann möglich, wenn die entsprechende Laborleistung zum Nachweis des Biomarkers auch im Versicherungskontext des Patienten erbringbar ist. Sind die entsprechenden Testverfahren ausreichend klinisch validiert, muss deren Abrechenbarkeit durch die Selbstverwaltung der Gesetzlichen Krankenversicherung zur Einführung des Arzneimittels gewährleistet werden, um den Anspruch des Patienten nicht leerlaufen zu lassen.

10. Die molekulare Therapie hat in der klinischen Entwicklung zahlreiche Besonderheiten: Die Entwicklung des Arzneimittels erfolgt am Rezeptormolekül, die Auswahl der Patienten für die klinische Prüfung knüpft am Vorliegen des Rezeptormoleküls oder anderer Biomarker an, die Subpopulationen sind oft klein und das Studiendesign in der Phase III der klinischen Prüfung kann es erfordern, dass Patienten mit einem Rezidiv mit dem (Prüf-)Arzneimittel behandelt werden (cross-over-design), so dass am Ende der Studie die klinischen Outcome-Parameter gleich sind. Dies lässt es geboten erscheinen, das bestehende Konzept der Nutzenbewertung des IQWiG (Methodenpapier Version 4.0) auf seine Anwendbarkeit hin zu überprüfen und gegebenenfalls zu ergänzen oder zu modifizieren. Molekulare Therapie und Evidenzbasierte Medizin stehen nicht in einem Gegensatz.

11. Die mit der Personalisierten Medizin verbundene Diagnostik kann zu prädiktiven Ergebnissen führen, die in der ge- sellschaftlichen Diskussion den Ruf nach individuellen, paternalistischen Vorgaben (,Nudging“) für eine Risikoprävention ergeben, die sich an genetischen Eigenschaften des Einzelnen orientiert. Gesundheitspolitische Bestrebungen, die Erkenntnisse der personalisierten Medizin zu Verpflichtungen oder Obliegenheiten des Einzelnen zu verdichten, sind als Verkürzung der Entscheidungsfreiheit des Einzelnen durch staatliche Vorgaben abzulehnen und weder rechtstaatlich begründbar noch verfassungsrechtlich zu legitimieren (vgl. hierzu „Einbecker Empfehlungen zu Genetischen Untersuchungen und Persönlichkeitsrecht der DGMR“", September 2002).

12. Die Grundlage für Prognosen und Behandlung von Krankheiten wird zunehmend von Daten determiniert, die zum einzelnen Patienten erhoben wurden. Eine neue, umfangreichere Verantwortung des Einzelnen für seine Gesundheit kann daran jedoch nicht geknüpft werden. Eine Durchsetzung von Verhaltensge- und Verboten mit dem „scharfen Schwert“ des Strafrechts muss ultima ratio bleiben und ist hier nicht angezeigt, und zwar mit Blick auf den Subsidiaritätsgrundsatz und die Autonomie des Einzelnen. Eine strafrechtliche Sanktionierung ,nicht gesundheitsbewussten Verhaltens" lässt sich daher nicht begründen.

Für das Präsidium der DGMR e.V.

Der Präsident

Rechtsanwalt Dr. A. Wienke

Fachanwalt für Medizinrecht

REZENSIONEN

DOI: 10.1007/s00350-013-3406-4

\section{Haftungsrecht und Entschädigungsfonds. Eine Unterschung zum deutschen und französischen Recht.}

Von Jonas Knetsch. Verlag Mohr Siebeck, Tübingen 2012, XXIII u. 257 S., kart., $€ 54.00$

Die Arbeit von Knetsch ist eine brillante, intra- und interdisziplinäre, rechtsvergleichende, teilweise empirische Untersuchung zum deutschen und französischen Recht, zu einem verhältnismäßig neuen, scheinbar abseitigen, an Relevanz zunehmenden Modell von Entschädigung und seinem Verhältnis zum Haftungsrecht, zur Systematisierung, zur Entwicklung und zur Perspektive von Entschädigungsfonds als Instrumenten der Bewältigung komplexer Schadenstypen. Sie ist zudem ein großer Gewinn für das Entschädigungsmanagement. Die Arbeit wurde in Deutschland und Frankreich mehrfach ausgezeichnet.

Die Arbeit besteht aus drei Teilen: Entschädigungsfonds als selbstständiges Modell kollektiver Schadenstragung (1), Funktionen von Fondslösungen innerhalb des sozialen Entschädigungsrechts (2) und Die Integration von Fondslösungen in das Gefüge des Entschädigungsrechts (3). Der umfangreichste Teil 1 gilt zunächst der Bedeutung von Fonds im deutschen und französischen Recht, stellt dann wichtige Fonds unterschiedlicher Art und Orientierung mit dem Schwerpunkt auf medizinrechtlichen Fonds in beiden Rechten und im internationalen Recht dar, um eine erste Typologie an ihren Funktionsweisen zu entwickeln. Die Information bezieht sich im deutschen Recht beispielsweise auf die Conterganstiftung, die Stiftung humanitäre Hilfe für durch Blutprodukte HIV-infizierte Personen, das Anti-D-Hilfegesetz, Fonds im Bereich des Umweltrechts, im französischen Recht beispielsweise auf den Fonds de garantie des assurances obligatoires de dommages (FGAO) und das Office nationale d'indemnisation des accidents médicaux, des affections iatrogènes et des infections nosocomiales (ONIAM). Eine wichtige

Prof. Dr. iur. Dieter Hart,

Bremen, Deutschland
Gründungsidee ist dort die ,solidarite nationale“. Unterschieden werden haftungsersetzende und -ergänzende, primäre und subsidiäre Fonds sowie Garantie-, Ergänzungs- und Kompensationsfonds. Es wird eine Systematik entwickelt, die zwischen retrospektiven (Regulierung von zurückliegenden Großschäden) und prospektiven (Instrument zur Lückenfüllung im Haftungsrecht) Fonds unterscheidet. Beide Typen werden in das Entschädigungsrecht eingeordnet, wobei eine Abgrenzung $\mathrm{zu}$ privatversicherungsrechtlichen und sozialversicherungsrechtlichen Instrumenten erfolgt und die Nähe (,Teilelement"; ,,autonomes Schadenstragungsmodell“) zum sozialen Entschädigungsrecht betont wird. Insgesamt handelt es sich um eine vorzügliche Analyse- und Systematisierungsarbeit, die von Ende zu Wende überzeugt.

Teil 2 systematisiert die Fondsfunktionen innerhalb des sozialen Entschädigungsrechts. Die beiden möglichen Funktionen der Fonds parallel zum Haftungsrecht, Ausgleich und Prävention werden prozess(Verfahrensgrundrechte) und materiellrechtlich (spezielle Schadenspositionen; Relativierung Totalreparation), aber auch psycho-soziologisch (Geschädigter und Viktimologie; Beteiligungsrechte) analysiert, wobei insbesondere der Aktivierung der Präventionsfunktion der Fonds - eine typische Leerstelle - breiter Raum eingeräumt wird.

Der dritte Teil gilt der Integration von Fondslösungen in das System des Entschädigungsrechts. Unter diesem Titel werden die Beziehungen von Fonds zum Haftungsrecht (Zusammentreffen von Fonds und Haftungsrecht; Ergänzung, Ersetzung und Kumulierungsverbot) und die Koordination von Fonds untereinander (Zusammenführung versus differenziertes Entschädigungsrecht als Ausdruck unterschiedlicher kollektiver Verantwortlichkeiten) behandelt, wobei letzteres dem Gegenstand geschuldet fast nur das französische Recht betrifft.

Die drei Teile Recht, Funktionen und Integration der Fonds sind ein ausgezeichnet integriertes Gesamtwerk, das einen wertvollen Überblick mit Detailanalysen, eine überzeugende Systematisierung von Fondsmodellen mit rechtpolitischen und Managementperspektiven der Gestaltung von Entschädigungssystemen verbindet. Es handelt sich sowohl thematisch wie formal, sachlich und in der Durchführung um eine außergewöhnlich lobenswerte Dissertation. Jeder, der rechtlich, rechtspraktisch wie rechtstheoretisch und rechtspolitisch mit Entschädigungsfonds zu tun hat, muss diese Arbeit lesen. Für andere haftungsrechtlich Interessierte ist die Lektüre ein großer intellektueller Gewinn. 